\title{
Passenger Protection Performance of Breakaway Type Posts Made of High Anti-Corrosion Steel Materials
}

\author{
Sang-Youl Lee ${ }^{1} \cdot$ Byung-Jik Son ${ }^{2} \cdot$ SeongHyeok Lee ${ }^{3} \cdot$ WooYoung Jung ${ }^{4}$
}

\begin{abstract}
A finite element simulation study was performed to gain an insight about the crash test details. Accuracy of the simulation was verified using qualitative and quantitative comparisons. Based on in-depth examination of crash simulation recordings, energy distributions occurred in the breakaway type posts against the car are determined. The numerical results for various parameters are verified by comparing different material models with internal energy distribution occurred in the breakaway type posts from the crash simulation.
\end{abstract}

Keywords- breakaway type posts, anti-corrosion steel, passenger protection, finite element analysis

\section{Introduction}

Street lights or sign board posts, which are standard roadside structures, are essential elements for the safe passage of vehicles and pedestrians. They are designed to withstand wind loads because of their function as auxiliary roadside structures. However, in terms of automobile crashes, they are hazardous elements on the road. In sites where there are no guard rails on the roadside, a crash into such a post will cause the vehicle to absorb much of the impact energy, significantly endangering the passengers of the cars. To avoid this problem, a recent study conducted in Korea assessed passenger safety for coupled, rounded posts. In this study, we used LS-DYNA software to perform a simulation with high anti-corrosion breakaway posts capable of absorbing impacts and calculated the breakage stress to assess passenger safety. Passenger safety was assessed by calculating the THIV and PHD. This process compared normal steel material (SS400) and high anticorrosion steel material.

\footnotetext{
${ }^{1}$ Sang-Youl Lee/Associate Professor/Ph.D.

Department of Civil Engineering, Andong National University

Andong, South Korea

${ }^{2}$ Byung-Jik Son/Associate Professor/Ph.D.

Department of Civil Engineering, KonYang University

NonSan, South Korea

${ }^{3}$ SeungHyeok Lee/Researcher/Ph.D.

Korea Railroad Research Institute (KRRI)

Uiwang, South Korea

${ }^{4}$ Woo-Young Jung/Professor/Ph.D.

Department of Civil Engineering, Gangneung-Wonju National University

Gangneung, South Korea
}

The goal of this study was to assess passenger safety in a car impacting the post. For passenger safety assessment, the THIV requirement was $33 \mathrm{~km} / \mathrm{h}$ or lower, while the PHD requirement was 20 or less to ensure safety of the passenger. For this reason, we used a coupled post modeling to assess the risks to which passengers are exposed.

\section{Mechanical interpretation of the impact breakaway}

If two blocks, namely $\Omega_{\mathrm{A}}$ and $\Omega_{\mathrm{B}}$ collide with block element $\Omega \mathrm{C}$ at a velocity of $\mathrm{v}$, the process is as shown in Figure 1. Assuming that blocks $\Omega_{\mathrm{A}}$ and $\Omega_{\mathrm{B}}$ are completely attached without any gaps, the force at the interface generated in the finite elements after the crash into block $\Omega_{\mathrm{C}}$ can be divided into the vertical force $f_{n}$ and the shearing force $f_{t}$. In normal crash interpretations, the vertical force is higher than the shearing force. Especially when one considers an impact breakaway, the vertical force created inside the finite element occurs at the interface due to the momentum of the post.

To understand the mechanical concepts, we used LSDYNA and the TIEDBREAK_NODES_ONLY option to simulate the distribution of the force on the surface of block $\Omega_{\mathrm{A}}$ in a full contact. (Figure 2-4) The bottom of block $\Omega_{\mathrm{A}}$ was fixed, and blocks $\Omega_{\mathrm{A}}$ and $\Omega_{\mathrm{B}}$ were assumed to be in complete contact. Block $\Omega_{\mathrm{C}}$ proceeded in the $-\mathrm{x}$ direction at $0.1 \mathrm{~m} / \mathrm{s}$ to crash into block $\Omega_{\mathrm{B}}$. Figure 5 shows the graphs of the forces in the finite elements of $\Omega_{\mathrm{A}}$, indicating the vertical and shearing forces. In this simulation, we used simple block models and the point of impact was lower than the center of gravity. This caused the shearing force to become higher. Concerning the post, it is the vertical force that affects the breakaway most significantly. Therefore, we decided to ignore the influence from the shearing force. The maximum vertical force was $2.1 \mathrm{~N}$ at 3.83 seconds, and we applied the impact breakaway under the following conditions. For this reason, among the LS-DYNA input TIEDBREAK_NODES_ONLY options NFLF(Normal failure force) was $1 \mathrm{~N}$, while $\mathrm{SFLF}$ (Shear failure force) was $1 \mathrm{e} 6 \mathrm{~N}$.

$f n<f n-\max =2.1 \mathrm{~N}$ (Maximum normal force) 
Figures 6 and 7 show the behavior of the blocks after the impact when we applied the vertical force breakaway conditions as set forth above. Figure 8 shows the distribution of the shearing stress in the block $\Omega_{\mathrm{A}}$ element. All vertical forces are within the range of $1 \mathrm{~N}$. What this tells us is that, when the blocks are detached, the maximum vertical force is calculated under full contact. A failure force of smaller value causes the detachment of the elements .

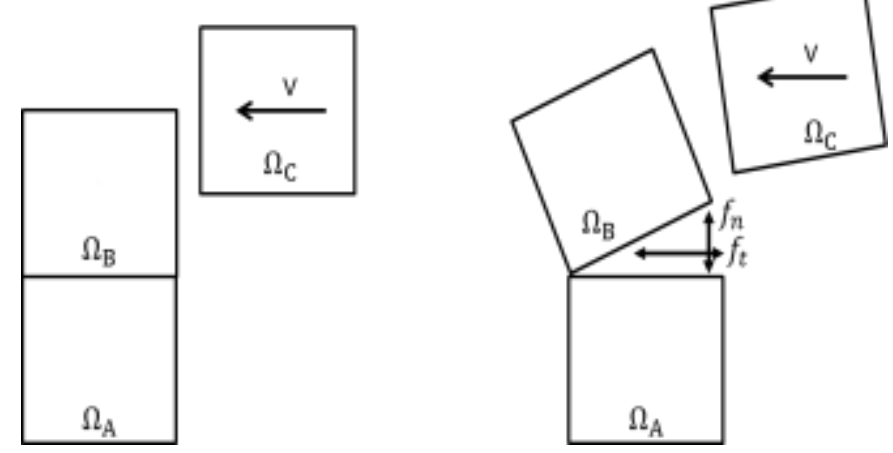

Figure 1. Detachment of the blocks due to impact
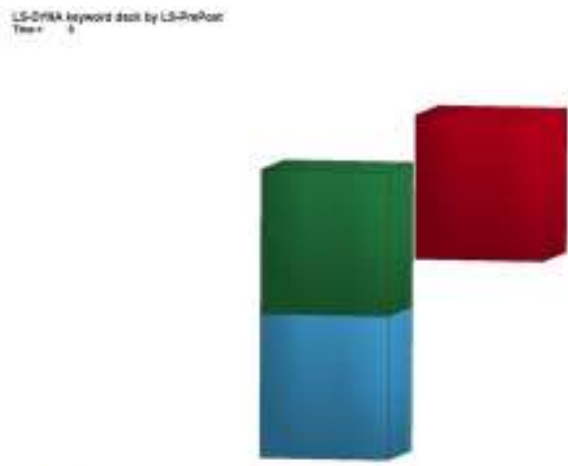

Figure 2. Block impact under full contact conditions $(0 \mathrm{sec})$

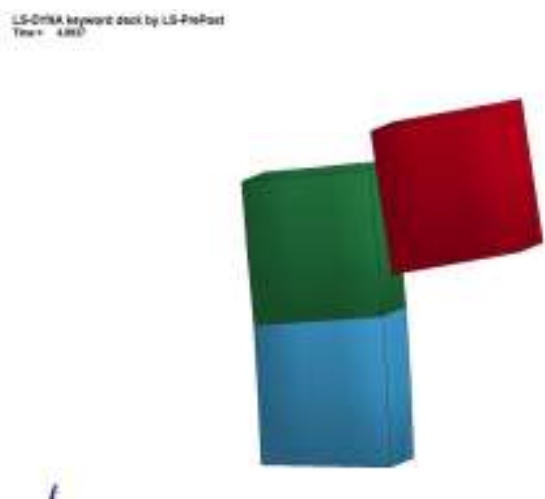

Figure 3. Block impact under full contact conditions $(4.8 \mathrm{sec})$

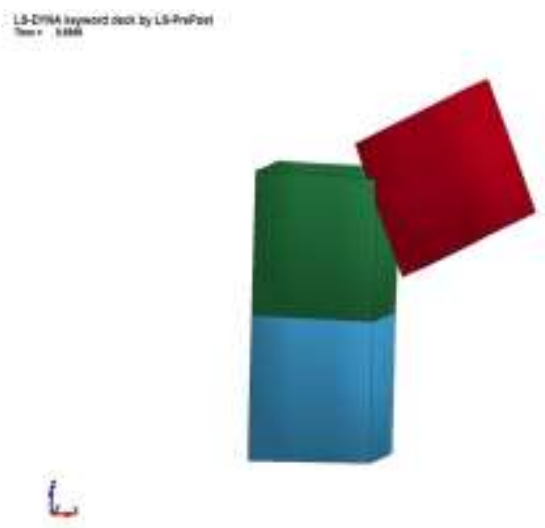

Figure 4. Block impact under full contact conditions

$(9.8 \mathrm{sec})$

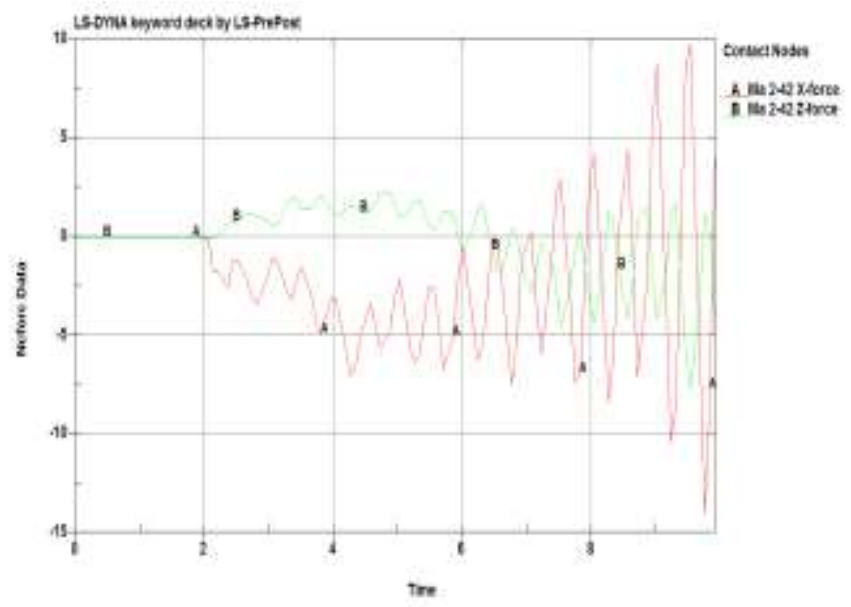

Figure 5. The distribution of the vertical and shearing force in block element $\Omega_{\text {A }}$ ( $\mathrm{z}$ axis: vertical force, $\mathrm{x}$ axis: shearing force)
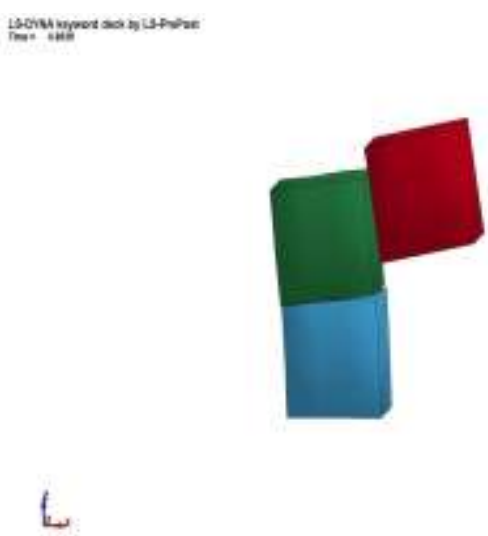

Figure 6. Block impact under breakaway conditions in the equation (1)

$(4.8 \mathrm{sec})$ 


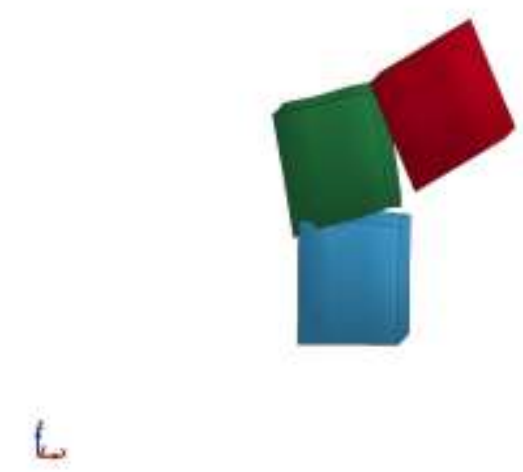

Figure 7. Block impact under the breakaway conditions in the equation (7) $(9.8 \mathrm{sec})$

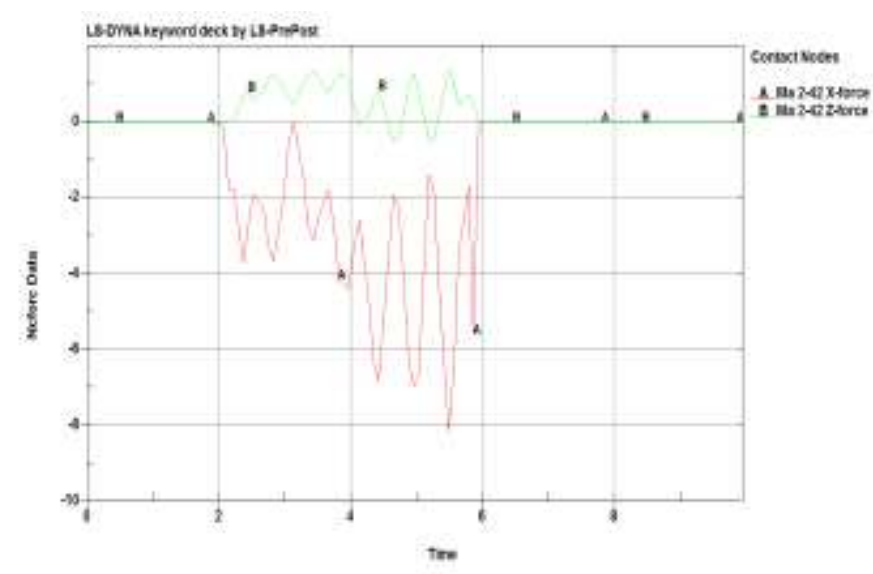

Figure 8. The distribution of the vertical and shearing force in block element $\Omega_{\mathrm{A}}$ in consideration of the breakaway conditions ( $\mathrm{z}$ axis: vertical force, $\mathrm{x}$ axis: shearing force)

\section{Crash Interpretation Modeling}

The breakaway post is deformed when a car crashes into it. It is designed to breakaway when the threshold stress value is exceeded during impact. The height of the post used for this study was $3,200 \mathrm{~mm}$ for the upper post and $500 \mathrm{~mm}$ for the lower post, totaling $3,700 \mathrm{~mm}$. The lower post is buried under the ground and modeled to be coupled but with freedom in 6 directions. (Figure 9) $\mathrm{We}$ also assumed that there is a signboard that is $1,250 \mathrm{~mm} \times 1,200 \mathrm{~mm}$ and completely attached to the post. The upper and lower posts were modeled to breakaway once the threshold failure force is reached. The steel pipe post modeled had a diameter of $101.6 \mathrm{~mm}$ and a wall thickness of $4 \mathrm{~mm}$. It was modeled as a shell element. The same parameters were given to the sign board, which was modeled as a shell element with $4 \mathrm{~mm}$ wall thickness. The 4 facing clips and 2 slip bases were built in solid elements. Also, since the purpose of this project was to compare the safety of passengers with posts built with SS400 and those built with SM490, each of these materials was applied to the post and the results compared. The material mode was Cowper-Symonds, with a view to consider the dynamic effect of the vehicle.

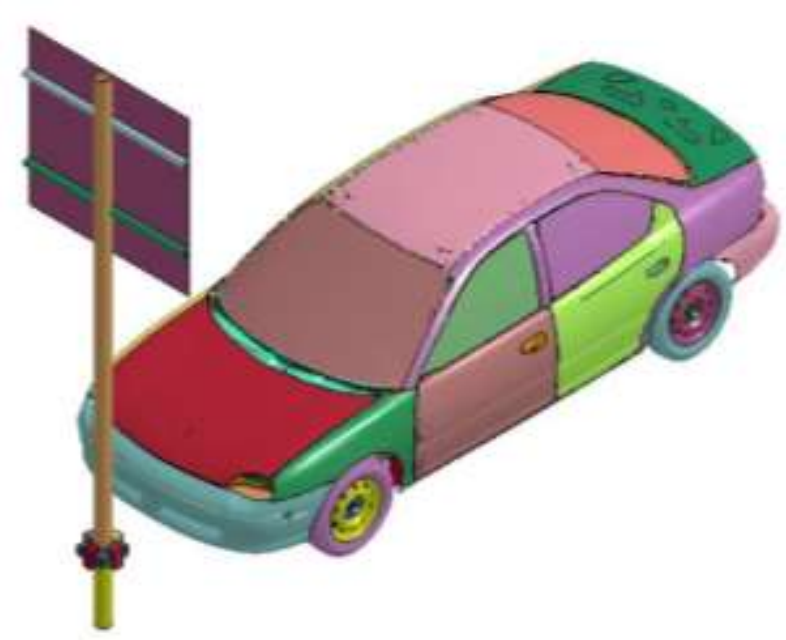

Figure 9. Breakaway type post mode using shell and solid elements

A Dodge-Neon was chosen for the passenger vehicle model as provided by NCAC, the National Crash Analysis Center of the United States. The physical properties of most of the elements in the vehicle were yield stress $400 \mathrm{MPa}$ and elastic coefficient of 210,000Mpa. The impact interpretation between the post and vehicle were limited by the Contact options in the LS-Dyna software.

The crash simulation in LS-DYNA begins basically with the Explicit time interpretation. In case of Explicit, there is a problem that the user has to define an arbitrary time span. Narrower spans produce lower accuracy results. In this simulation, the contact conditions were very complicated. Therefore, the automatic time increment option supported by LS-DYNA was used. In the end, a total of 18 cases were simulated. Of these, 9 had the posts built with SS400, while the rest had the posts built with SM490. We assumed the wall thickness of the posts to be the same to determine the conditions of breakaway by material. The interpretations of each case in accordance with the breakaway conditions in equation 1 are shown in Table 1.

TABLE 1. Interpretations by breakaway conditions (Breakaway conditions $f_{t-\max }(\mathrm{N})$ )

\begin{tabular}{|c|c|c|c|c|c|c|c|c|c|}
\hline \multirow{2}{*}{55400} & Case0 & Case1 & Case2 & Case3 & Case4 & Case5 & Case6 & Case7 & Case8 \\
\cline { 2 - 9 } & 199 & 5000 & 10000 & 15000 & 20000 & 25000 & 30000 & 40000 & 50000 \\
\hline \multirow{2}{*}{ SM490 } & Case9 & Case10 & Case11 & Case12 & Case13 & Case14 & Case15 & Case16 & Case17 \\
\cline { 2 - 9 } & 109 & 5000 & 10000 & 15000 & 20000 & 25000 & 30000 & 40000 & 50000 \\
\hline
\end{tabular}




\section{Estimation of THIV and PHD}

Figures 10 and 11 show graphs of THIV and PHD as drawn by the TRAP program after measuring the vehicles acceleration in $\mathrm{x}, \mathrm{y}$, and $\mathrm{z}$ axis as well as the rotational velocity. Assessments of the THIV and PHD were performed based on the passenger safety performance assessment. In the case of THIV, passenger safety could be guaranteed when the speed was $33 \mathrm{~km} / \mathrm{h}$. In this project, the requirement was satisfied for both SS400 and SM490, within the failure force range of $40,000 \mathrm{~N}$. However, in the case of PHD, the SS400 satisfied the requirement under $50,000 \mathrm{~N}$, while SM490 did so under $40,000 \mathrm{~N}$. If we relate this to the breakage results discussed above, the SS400 is believed to have satisfied the THIV requirement after the breakaway, while SS490 satisfied the THIV requirement in case no. 15 , where the breakaway did not occur. The same is true with the PHD. In other words, the condition that both the THIV and PHD should be satisfied for SS400 and SM490 was that the failure force is lower than $40,000 \mathrm{~N}$.

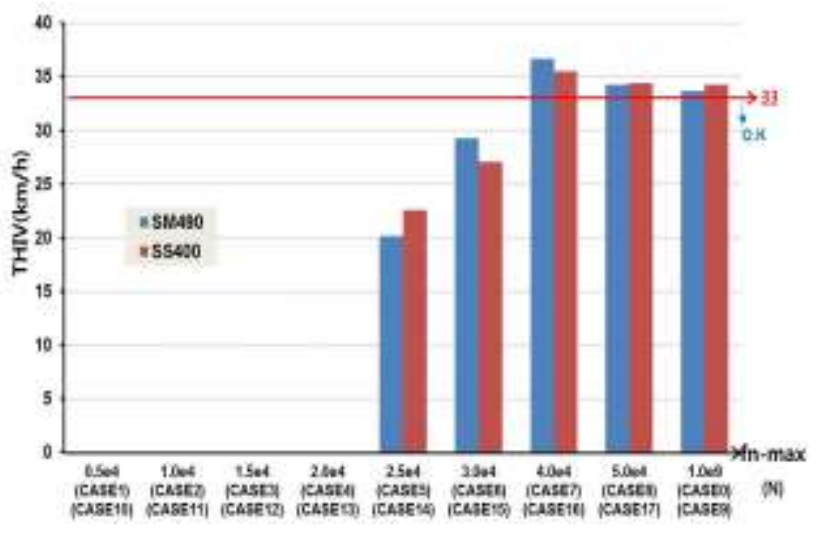

Figure 10. THIV by cases

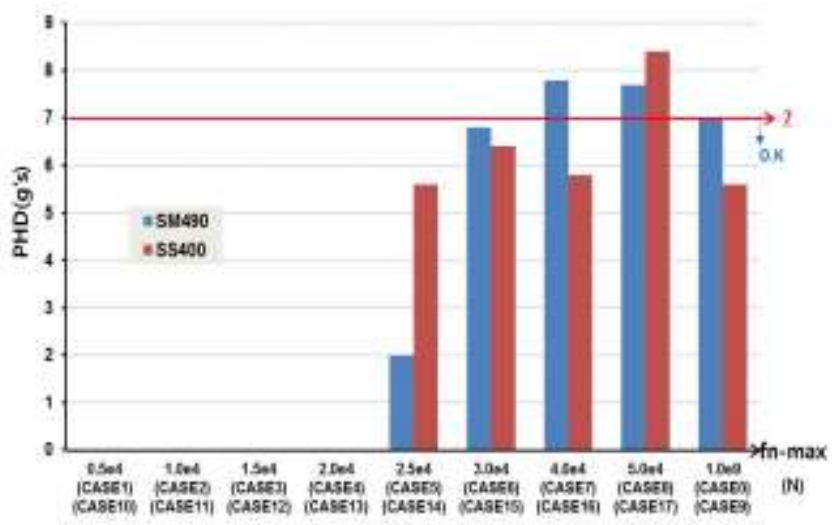

Figure 11. PHD by cases

\section{v. Conclusion}

In this study, we performed the assessment of passenger safety for posts and signboards that are standard roadside installations. To disperse the impact force when a car crashes into a post, the post was designed with a breakaway feature. The simulation was performed with two different materials-normal steel (SS400) and high anti-corrosion material (SM490.) We determined that previous studies did not provide sufficient explanations of the mechanism of breakaways. Therefore, this study was performed to clarify the conditions of post breakaway. With the maximum vertical force as the basis, 18 cases were considered. The results showed that the SS400 material had a higher breakaway requirement compared to that of SM490, and we believed that such difference was related to the physical properties of the steel material used for the post. The assessment results of the passenger safety for THIV and PHD showed that the requirement was satisfied under 40,000N for both the SS400 and SM490 materials. However, in the case of SM490, while the post was not detached, both the THIV and PHD figures showed satisfactory results.

\section{Acknowledgment (Heading 5)}

This work was supported by the National Research Foundation of Korea (NRF) grant funded by the Korea government (MSIP) (No. 2015R1A2A2A01005637) and also supported by a grant from $R \& D$ Program of the Korea Railroad Research Institute, Republic of Korea..

\section{References}

[1] AASHTD (2009), Manual for Assessing Safety Hardware.

[2] M.A.Crisfield (1997), Nonlinear Finite Element Analysis of Solids and Structures, Wiley.

[3] Cowper, G. and Symonds, P. (1957), Strain Hardening and Strain Rate Effects in the Loading of Cantilever Beams, Brown Univ. Applied Mathematics Report, Report No. 28.

[4] Johnson, G.R. and Cook, W.H. (1985), "Fracture Characteristics of Three Metals Subjected to Various Strain, Strain Rates Temperatures and Pressures", Engineering Fracture Mechanics, 21(1), pp.31-48.

[5] Test Risk Assessment Program (TRAP) Version 2.0 1999, User's Manual.

[6] Bowels, J.E. (1977), Foundation Analysis and Design, Second Ed., McGraw-Hill.

[7] National Crash Analysis Center, http://www.ncac.gwu.edu

[8] ANSYS(LS-DYNA) User's Manual, 2012, ANSYS Corp. 